\title{
1 Nestmate recognition of early brood in ants
}

2

3 Arthur de Fouchier ${ }^{1,4}$, Chloé Leroy ${ }^{1}$, Abderrahman Khila ${ }^{2}$, Patrizia d'Ettorre ${ }^{1,3}$

$4{ }^{1}$ Laboratoire d'Ethologie Expérimentale et Comparée, Université Sorbonne Paris Nord, Villetaneuse, 5 France

$6 \quad{ }^{2}$ Université de Lyon, Institut de Génomique Fonctionnelle de Lyon, CNRS UMR 5242, Ecole Normale

7 Supérieure de Lyon, Université Claude Bernard Lyon 1, Lyon, France

$8{ }^{3}$ Institut Universitaire de France (IUF), Paris, France

$9{ }^{4}$ Present adress: Institut d'Ecologie et des Science de I'Environnement de Paris, INRAE, Sorbonne

10 Université, CNRS, IRD, UPEC, Université Paris Diderot, Paris, Versailles, France

Ants, Brood retrieval, Cuticular hydrocarbons, Nestmate recognition, Social parasitism

\section{Abstract}

Brood is critically important in social insect colonies. It carries the colony fitness through delivering future reproductive adults as well as workers that will increase the colony's workforce. Adoption of non-nestmate brood can be a mean to increase colony's workforce but entails the risk of rearing unrelated sexuals or social parasites. For early brood (eggs and L1 larvae), this balance is less positive as young brood need a substantial amount of resource before becoming workers. Thus, it appears beneficial for ant workers to discriminate between nestmate and alien brood using the chemical cues displayed at the brood's surface. However, the chemical signature of ant early brood stages and its use by workers remains understudied. To fill this gap, we investigated the chemical basis of early brood nestmate and cross-species recognition in six Formicoid ants. We also tested the discrimination behaviour of workers in brood retrieval trials. We observed clear species-level cues and discrimination against heterospecific brood. We also found that eggs and most young larvae display a colony signature but that only some species discriminate against non-nestmate eggs and L1 larvae. Interestingly, these species appear to also be those belonging to genera subject to brood parasitism. 


\section{Introduction}

In ants, workers are fully or partially sterile $[1,2]$. Workers achieve fitness indirectly by rearing their mother's brood to provide workforce and future reproductive individuals (males and queens). This reproductive division of labour is a hallmark of highly social societies and place brood at the centre of ant colonies. Workers promptly retrieve eggs and larvae found outside the nest [3], and secure them in case of colony disturbance [4]. Behavioural studies have shown that ant workers adopt brood from other nests, and even other species, while keeping a preference for nestmate eggs and larvae [5].

Brood adoption is an adaptive behaviour as larvae raised in a foreign and unrelated nest may eventually integrate the colony workforce $[6,7]$. Incipient colonies of Lasius niger and Messor pergandei often raid brood from close-by colonies to increase their chance of survival [8]. Brood theft can also take place during nests relocation [9]. However, adopting non-nestmate brood entails a risk. Some ant species are subject to social parasites, such as inquilines and slave-makers, which take advantage of the host workers to raise their own brood and have a clear negative impact on the fitness of their host colony. [10,11].

In theory, adopting non-nestmate brood involves a trade-off, for ant workers, between the gain of future workforce and the potential cost of raising unrelated reproductive individuals or accepting a social parasite [7]. It seems thus adaptive to develop counter-measures to avoid such risks. Among the possible adaptations, there is the ability of workers to recognize intruding non-nestmate adults and brood [12], as occurs in populations subjects to brood parasitism [13]. However, adaptations at the species level are not well understood $[10,11]$.

Ants are usually efficient in recognising non-nestmates and behave aggressively toward competitors for the resources of the environment [14]. Nestmate recognition relies on the detection of colonyspecific chemosensory cues. These are long chain hydrocarbons found on the outer surface of developing and adult individuals. The hydrocarbons can be linear, saturated or unsaturated, or contain methyl groups (methyl-branched alkanes) $[15,16]$. The blend of hydrocarbons displayed by each individual is the results of both genetic [16] and environmental factors [17]. Consequently, members of the same colony, which are often related and live in the same environment, share similar hydrocarbon profiles. Cuticular hydrocarbons homogenize between members of the colony through mutual grooming, food sharing, inter-individual contacts or contact with the nest-material $[16,18]$.

The importance of brood nestmate recognition for ant colonies led to 40 studies in 33 ant species (as reviewed in [5]). However, those studies focused mostly on mid to late-stage larvae, while early brood stages remain understudied. Hydrocarbons displayed on ant eggs have been studied in few genera [19-25]. To our knowledge, a colony-level signature of the surface hydrocarbons of the eggs has been convincingly found in two genera, belonging to the Ponerinae and the Formicinae [21,25].

Eggs can acquire the hydrocarbon signature through various mechanisms. The source of colony-level cues on brood is a question better studied in eggs than larvae. Freshly deposited eggs already bear the colony signature [25]. Mothers appear to deposit hydrocarbons on eggs while they are maturing in their ovaries $[20,26]$. Once laid, eggs surface hydrocarbons could be influenced by contact with workers and allo-grooming [16,27]. However, the effect of contact alone is probably not a rapid process [28]. It is possible that embryos produce hydrocarbons that might traverse the chorion through pores and modify the egg surface hydrocarbons [29].

Surface hydrocarbons and nestmate recognition of early stage larvae remains critically understudied. When larvae hatch from their egg, it is unclear if the surface hydrocarbons are transferred to the 
74 larvae or if freshly hatched larvae shall de novo synthesize surface hydrocarbons [30]. In 75 Aphaenogaster senilis, the quantity of surface hydrocarbons on larvae is smaller compared to eggs 76 and workers [31]. Most of the hydrocarbons on the surface of eggs are likely not transferred to the 77 larvae. As such, whether fist instar larvae display enough cues to be recognised as nestmate remains 78 an open question.

79 In this study, we aimed at filling the gap in our knowledge of nestmate recognition of early brood 80 stages in ants. We investigated the colony-level signature of surface hydrocarbons of eggs and first 81 instar (L1) larvae from six species belonging to three different subfamilies of the Formicoid clade 82 [32]: Myrmicinae, Formicinae and Dolichoderinae. To assess how selective workers are when 83 adopting brood, we studied brood retrieval behaviour of workers facing eggs and L1 larvae 84 originating from their colony (nestmate), from another homospecific colony (non-nestmate) or from another species (heterospecific). 


\section{Material and Methods}

For complete details on the materials and methods, see Supplementary Material and Methods.

\section{Ant colonies}

We used colonies of six ant species: Aphaenogaster senilis (Formicidae, Myrmicinae), Camponotus aethiops (Formicidae, Formicinae), Formica fusca (Formicidae, Formicinae), Lasius niger (Formicidae, Formicinae), Messor barbarus (Formicidae, Myrmicinae) and Tapinoma darioi (Formicidae, Dolichoderinae) housed in the laboratory.

\section{Chemical analyses}

We collected at least three eggs and first instar larvae from at least three different colonies for each of the six species. Surface hydrocarbons where extracted from eggs and larvae using $10 \mu \mathrm{l}$ of $\mathrm{n}$ pentane ( $\geq 99 \%$, HPLC grade, Sigma-Aldrich) for 2 minutes. We then injected $3 \mu \mathrm{L}$ of the extract into an Agilent 7890A gas chromatograph (GC) coupled to an Agilent 5975 C mass spectrometer (MS).

\section{Behavioural experiments}

The aim was to test the behaviour of workers when facing nestmate, homo-specific non-nestmate or hetero-specific eggs or first instar larvae. The same protocol was followed for eggs and L1 larvae trials, which were performed independently. Brood and workers originated from twelve A. senilis colonies, ten $C$. aethiops, Lasius niger and M. barbarus colonies and six T. darioi colonies. Six F. fusca colonies were used as source of hetero-specific brood. We prepared groups of six nestmate workers (three from outside the nest and three from inside the nest) in an eight $\mathrm{cm}$ arena with a filter paper as floor and with walls coated with Fluon ${ }^{\circledR}$ (AGC Chemicals Europe, Thornton-Cleveleys, United Kingdom). Each group was given a refuge made of a red-coated $1.5 \mathrm{~mL}$ Eppendorf tube (that had spent at least twenty-four hours in the box of the original colony), three late-instar larvae from their own colony, food (mixture of honey and apple) and water. After twenty-six hours of acclimation, if the workers had brought the late-instar larvae into the refuge, we removed food and water and started the behavioural trials.

Shortly before the trials, we collected eggs or L1 larvae from the colony of origin of each groups of workers (nestmate), from another colony of the same species (non-nestmate) or from another species (hetero-specific). For heterospecific brood, we used brood from species of the same subfamily and of similar size when available. For $A$. senilis, we used M. barbarus brood and vice versa. For $C$. aethiops and $L$. niger, we used $F$. fusca brood. For T. darioi, we used $L$. niger brood. For each trial, three brood items were deposited in a line (Supplementary Figure S1). All three of these were either nestmate, or non-nestmate or heterospecific relative to the workers. The behaviour of the workers towards the brood items was video recorded with an FDR-AX33 Sony camera for fifteen minutes. After fifteen additional minutes, any brood that had not been brought inside the refuge were removed from the arena. Thirty minutes later, another set of three brood items with a different origin were presented to the same group of workers. This was repeated three times. Therefore, each group of workers received nine brood items in total in 3 different trials of all the three possible origins. The order of presentation of the type of brood items was controlled to prevent any bias.

The behaviour of the workers was scored for the first 15 minutes after the first brood item was deposited using the software Boris v7.9.15 [33]. We noted the times where workers started and stopped to antennate a brood item and the times when a worker entered the refuge with a transported brood item. Trials for which the workers did not enter in contact with the brood items were discarded from further analysis as workers were considered inactive. 
130 Data and statistical analyses

131 Data was analysed using R Studio (v1.3.1093, RStudio Team, 2015) and R software (v4.0.0, R Core

132 Team, 2020).

\section{Chemical data}

134 For each colony and species, we analysed between three and four samples. We selected peaks that 135 were present in all the samples of the same species. We integrated the area of each peak and 136 normalised it to the sum of the area of all peaks in a given sample. We then did a principal 137 component analysis (PCA) for each species, keeping enough components to describe at least $95 \%$ of the total variance. Using components with an F-score, relative to the colony of origin, superior or equal to 0.01 , we computed linear discriminant analysis for each species and brood types separately using the colony of origin as classification variable with a leave-one sample out cross-validation. To test the significance of the accuracy of classification obtained, we used permutation tests with 5000 simulations.

To assess the variability of the difference between nestmate and non-nestmate chemical signatures across species, we computed intra and inter-colony Euclidian distance between nestmates and nonnestmates using the global centroid method [36]. To assess the variation of intra-colony distances between species, we computed the ratio between intra and inter-colony distances. We then performed type II ANOVA on linear mixed-effects models (LMM) of the effect of the species of origin of the samples on the ratios of the intra and inter-colony chemical distances. Sample ID and colony of origin were used as nested random factors. The colony used for the inter-colony distance was a random factor as well. P-values were adjusted for multiple comparisons across species for each type of brood using Holm's method.

\section{Behavioural data}

We tested whether the source of the brood item had an effect on two different variables: 1) the number brood items brought into the refuge in each trial; 2) the total time workers spent antennating the brood items. The number of brood items brought to refuge was analysed using generalised linear mixed-effect models (GLMM). For the cumulative duration of antennation, we used LMMs. The colony of origin of the workers, their group identity, the origin and the order of the brood encountered during the three trials were used as random factors. Post hoc differences were 
160

161

162

163

164

165

166

167

168

169

170

171

172

173

174

175

176

177

178

179

180

181

182

183

184

185

186

187

188

189

190

191

192

193

194

195

196

197

198

199

200

201

202

203

204

\section{Results}

\section{Egg surface hydrocarbons}

In the extracts of egg surface compounds, we could observe between 21 (A. senilis and L. niger) and 31 (C. aethiops) peaks containing hydrocarbons that were consistently present in samples of the same species (Figure 1, Supplementary Figure S2). These profiles contained a majority of methylalkanes and a smaller proportion of alkanes. In T. darioi, L. niger and F. fusca egg samples, we also observed a small proportion of alkenes. (Figure 1.A). The chemical profile of larvae had a lower quantity of hydrocarbons compared to eggs (Figure 1.B) and a smaller diversity of compounds (Figure 1.A). We found between 5 (in L. niger and F. fusca) and 9 (in C. aethiops) peaks containing hydrocarbons with a majority of alkanes and a lower proportion of methyl-alkane in almost all species. In $M$. barbarus, both families of compounds were present in similar numbers (Figure 1.B). We did not observe any alkenes among the surface hydrocarbons extracted from larvae. The most common compounds are $C_{23}, C_{25}$ and $C_{27}$ (peaks 4,21 and 45), which are present across all species in surface profiles of both eggs and larvae (Figure 1.C, Supplementary Table S1). The alkane $C_{28}$ (peaks 59) was found in all egg samples. In almost all cases, compounds found in L1 larvae extracts were also present in eggs exacts (Figure 1.C, Supplementary Table S1). The only exception is a diMeC $\mathrm{C}_{24}$ (peak 15) found on $A$. senilis larvae.

Principal component analyses indicate that there is a colony-specific blend of surface hydrocarbons, (Supplementary Figure S3, Supplementary Table S2). Using a linear discriminant analysis, we observed that chemical profiles allowed the prediction of the colony of origin of the samples significantly better than by chance for all the egg samples (permutation test, $p \leq 0.05$, Figure 2.A, $[37,38])$. The accuracy of prediction of the colony of origin was $100 \%$ for L. niger, C. aethiops, F. fusca and $M$. barbarus eggs. For $T$. dairoi and $A$. senilis eggs, the prediction of the colony of origins was not completely accurate $(88.89 \%$ and $93.33 \%$ respectively). In larvae samples, the hydrocarbon profiles allowed the identification of the colony of origin in L. niger, C. aethiops, F. fusca and M. barbarus (permutation test, $p \leq 0.05$, Figure 2.A). However, unlike for egg samples, the accuracy of prediction of the colony of origin was $100 \%$ only for C. aethiops and F. fusca. Regarding M. barbarus and L. niger L1 larvae, the predictions were accurate around two thirds of the time. For $T$. darioi and A. senilis L1 samples, prediction of the colony of origin was inaccurate and not different from random.

To compare the difference between colony hydrocarbon profiles across species, we normalised the nestmate chemical distances relative to the non-nestmate distances in each species (Figure2.B). The difference in colony signatures are similar for larvae and for eggs in most species. However, in $L$. niger and $F$. fusca eggs differences in colony signatures are larger compared to $T$. darioi, $C$. aethiops and $M$. barbarus nestmate to non-nestmate distances (LMM, $\mathrm{p} \leq 0.05$, Type II ANOVA; Supplementary Table S3). Consistently with our analysis of the existence of a colony signature in the chemical profiles of eggs, the large majority of ratios between nestmate and non-nestmate eggs chemical distances are inferior to one (i.e. distance between nestmates is smaller than between nonnestmates). For larvae, cases of ratios superior to one (i.e. distance between nestmates is greater than between non-nestmates) appear more frequently, which is consistent with our observations that colony signatures are either absent or less clear on L1 larvae.

\section{Brood discrimination by ant workers}

From the results of our chemical analysis, we would predict that ant workers are able to discriminate between homo-specific and hetero-specific brood. The discrimination between nestmate and nonnestmate would be possible for eggs but more difficult for L1 larvae, especially in $A$. senilis and $T$. 
205

206

207

208

209

210

211

212

213

214

215

216

217

218

219

220

221

222

223

224

225

226

227

228

229

230

231

232

233

234

235

236

237

238

239 darioi. Using behavioural assays, we measured the number of brood items retrieved by workers (Figure 3.A) as well as the time they spent antennating the brood (Figure 3.B).

For $T$. darioi, nestmate eggs were retrieved significantly more frequently compared to hetero-specific items (GLMM, $p \leq 0.05$, Type II ANOVA; Supplementary Table S4). We observed no differences in the number of non-nestmate and hetero-specific eggs retrieved by $T$. darioi workers. L. niger workers brought significantly more nestmate eggs into the refuge compared to non-nestmate and heterospecific eggs (GLMM, $p \leq 0.05$, Type II ANOVA; Supplementary Table S4). The number of nonnestmate eggs retrieved by $L$. niger workers was higher than the number of hetero-specific ones. The results for $A$. senilis, $C$. aethiops, L. niger and $M$. barbarus assays were similar: workers transported significantly more nestmate and non-nestmate eggs than hetero-specific ones into the refuge (GLMM, $p \leq 0.05$, Type II ANOVA; Supplementary Table S4). There was no significant difference between the number of nestmate and non-nestmate eggs retrieved by workers.

Regarding L1 larvae, $T$. darioi workers retrieved significantly more nestmate L1 larvae than nonnestmate and hetero-specific ones. There were no differences in the, almost null, number of nonnestmate and hetero-specific larvae retrieved by $T$. darioi workers. Observations for $L$. niger, $A$. senilis, $C$. aethiops, and $M$. barbarus L1 larvae trials were similar. The number of nestmate and nonnestmate L1 larvae transported into the refuge by workers were similar and significantly higher than the number of hetero-specific L1 larvae. Overall, the results of the behavioural assays show that ant workers are able to discriminate between homo-specific and hetero-specific eggs and L1 larvae. Furthermore, we observed that $L$. niger and $T$. darioi discriminate between nestmate and nonnestmate eggs and only $T$. darioi workers discriminate between nestmate and non-nestmate L1 larvae.

Antennation allows ants to use their chemical and mechanical sensors to explore items. A longer antennation time is a sign of a higher interest or more complex identification of the item. A. senilis and $M$. barbarus workers spent significantly more time antennating nestmate and non-nestmate eggs compared to hetero-specific eggs (LMM, $p \leq 0.05$, Type II ANOVA; Supplementary Table S5). L. niger workers antennated for a significantly longer time nestmate and non-nestmate L1 larvae when compared to hetero-specific ones (LMM, $p \leq 0.05$, Type II ANOVA; Supplementary Table S5). For $C$. aethiops, antennation times where significantly shorter when comparing nestmate to non-nestmate and hetero-specific L1 larvae (LMM, p $\leq 0.05$, Type II ANOVA; Supplementary Table S5). Finally, A. senilis workers spent less time antennating nestmate and hetero-specific L1 larvae compared to nonnestmate larvae (LMM, $p \leq 0.05$, Type II ANOVA; Supplementary Table S5).

Overall, our behavioural trials show that ant workers discriminate between brood items from their colony and hetero-specific ones. However, discrimination between nestmate and homo-specific nonnestmate brood is evident only in L. niger and T. darioi. 


\section{Discussion}

Our chemical analysis and behavioural trials allow a better understanding of species and colony-level chemical cues in the early brood stages of Formicoid ants as well as the discriminatory behaviour that is dependent on those cues. The quantity and the diversity of cues displayed is clearly smaller in first instar larvae compared to eggs in all species studied. This supports the hypothesis that when larvae hatch from the egg the hydrocarbons are not transferred from the egg's chorion to the larval cuticle.

The hydrocarbons observed on the surface of egg and L1 larvae are similar to those found in adults $[15,39]$. As such, they should be detected by the sensory systems of all ant species [40]. Our chemical analysis clearly showed that the surface hydrocarbons of eggs and L1 larvae are different among species. The inter-specific differences allow ant workers to discriminate both eggs and larvae of their species from brood of a different species in all our behavioural trials. This is consistent with what has been observed for eggs in some Formica species [5,41].

Are ants able to recognise the colony of origin of conspecific eggs? We observed colony-specific blend of hydrocarbons on eggs, suggesting that the display of colony cues on eggs is a trait present across the Formicoid ants (three of the five subfamilies). This is consistent with observations in seven Formica species [25]. Despite the presence of colony-specific cues, only T. darioi and L. niger workers discriminated against non-nestmate eggs in our behavioural trials. Data from the literature show that F. fusca workers and larvae discriminate against non-nestmate eggs $[42,43]$. Interestingly, our results showed that discrimination against non-nestmate eggs is not consistently corelated with larger differences between nestmate and non-nestmate odours. This indicates that non-nestmate discrimination can rely both on clearer display of the colony of origin or more accurate recognition of the cues displayed.

Can workers recognise nestmate first instar larvae? Our chemical analysis and behavioural trials with L1 larvae draw a less clear picture than for eggs. Data in the literature are also scant. Larvae from both Formicinae species we studied ( $L$. niger and $C$. aethiops) and those from $M$. barbarus (Myrmicinae) display a colony-specific chemical signature. However, these signatures did not allow for reliable identification of the colony of origin in two species from different subfamilies $(M$. barbarus and L. niger). We could not demonstrate the presence of a colony signature in the surface hydrocarbons of $T$. darioi (Dolichoderinae) and A. senilis (Myrmicinae) larvae. Surprisingly, T. darioi workers were the only ones discriminating between nestmate and non-nestmate larvae, which indicates that the larvae do display enough cues for colony recognition. This means that $T$. darioi workers either act on chemical cues that our method of analysis could not detect or use nonchemical cues. However, to our knowledge, the literature does not support the hypothesis that workers use non-chemical cues (e.g. visual or auditory) for nestmate larvae recognition. As such, the hypothesis that $T$. darioi first instar larvae displaying a colony odour seems the most plausible.

We observed $A$. senilis and $C$. aethiops workers behaving differently when facing nestmate larvae compared to non-nestmate larvae (i.e. different antennation durations). Is this an indication that they are able to recognise nestmate $\mathrm{L} 1$ larvae from non-nestmate larvae? On C. aethiops $\mathrm{L} 1$ larvae, we could detect a colony-level chemical signature. We could not do so on $A$. senilis first instar larvae, but neither could we on $T$. darioi larvae despite the clear behavioural evidences that they do display a colony signature. Given the lower overall quantity of surface hydrocarbons on L1 larvae compared to eggs, the chemical cues displayed might challenge the olfactory detection system of ant workers and the presence of non-nestmate cues might appear ambiguous to them. The long antennation time observed would then be a sign of the ant's difficulty to recognize the signature. Similar hesitation has been observed for recognition of ambiguous colony cues on adults [44]. 
285 Taken together, our observations allow us to confidently state that workers recognise and favour 286 nestmate first instar larvae only for $T$. darioi. In the other species, the cues are either too challenging 287 to recognise for the workers or they just don't act on them. Discrimination against non-nestmate 288 eggs, doesn't implies favouring nestmate first instar larvae. This differences across stages in non289 nestmate discrimination probably arose from the differences in the quality and the diversity of the 290 chemical cues displayed as the surface of the brood. Unlike eggs, larvae likely have to synthesize the 291 chemical cues they display from the first day of their life.

292 Looking at our observations and those from the literature with a phylogenetic perspective supports 293 the hypothesis that egg surface hydrocarbons display sufficient information for ant workers to 294 discriminate nestmate from non-nestmate eggs across most of the ants' phylogenetic tree. The 295 predominance of non-nestmate eggs discrimination in the majority of the Formicoid ant subfamily 296 studied (2 out of 3 ) would indicates that the last common ancestor of Formicoid ants was 297 discriminating against non-nestmate eggs. Dinoponera quadriceps workers also favour nestmate eggs 298 [21]. The last common ancestor of Formicoids and Poneroids would have been also discriminating 299 against non-nestmate eggs, but these evolutionary hypotheses require more work to be supported.

300 The three Formicoid species that discriminate against non-nestmate eggs belong to genera prone to 301 social parasitism. Indeed, L. niger is host to various social parasites from the Lasius genus [11]. And 302 the Tapinoma genus is known to be subject to parasitism by Bothriomyrmex species $[10,11]$. 303 Furthermore, host species of the Formica genus also discriminate against non-nestmate eggs [41]. 304 Our results and those from the literature are thus in accordance with the hypothesis that higher non305 nestmate brood discrimination could arise from the arms race between social parasites and host 306 species [43]. The parasites trying to get themselves recognised as nestmates inducing a more strict 307 discrimination of eggs as a species level adaptation in hosts [13].

308 Discrimination can lead to costly errors [45]. Accordingly, the three species we studied which are not 309 subject to an arms race with social parasites do not discriminate against non-nestmate brood. Brood 310 adoption appears less risky in those non-host species while recognition errors (discarding of 311 nestmate brood) represent a potential loss to the colony's fitness. This would explain the reduction 312 or disappearance of the discriminatory behaviour against non-nestmate eggs. Identification of first 313 instar larvae, which do not display as much chemical cues as eggs, appears a more challenging task, 314 which prevents a stricter non-nestmate discrimination in most species even parasitized ones. Overall, 315 our results support the hypothesis that social parasites induce a selective pressure on host species, 316 which maintain the discrimination against non-nestmate eggs while non-host species are less 317 selective for brood retrieval. 


\section{Acknowledgements}

319 The authors would like to thanks Baptiste Piqueret and Swetashree Kolay for their commentaries on 320 the design of the experimental protocols and the data analysis. We also, would like to thank Sarah 321 Quemin and Laurène Ozeel for their help in the realisation of some behavioural experiments and 322 their analysis.

\section{Funding}

325 Arthur de Fouchier, Patrizia d'Ettorre and Abderrahman Khila contribution to this work has been 326 supported by the grant ANR-17-CE13-0008 SocioDev.

328 Data availability

329 Data used for the analysis performed have been deposited on FigShare: $33010.6084 / \mathrm{m} 9$.figshare.14303078 and 10.6084/m9.figshare.14304167. 


\section{References}

1. Khila A, Abouheif E. 2008 Reproductive constraint is a developmental mechanism that maintains social harmony in advanced ant societies. Proceedings of the National Academy of Sciences 105, 17884-17889. (doi:10.1073/pnas.0807351105)

2. Khila A, Abouheif E. 2010 Evaluating the role of reproductive constraints in ant social evolution. Philosophical Transactions of the Royal Society B: Biological Sciences 365, 617-630. (doi:10.1098/rstb.2009.0257)

3. Lenoir A. 1981 Brood retrieving in the ant, Lasius niger L. Sociobiology 6, 153-178.

4. Meudec M. 1978 Response to and transport of brood by workers of Tapinoma erraticum (Formicidae; Dolichoderinae) during nest disturbance. Behavioural Processes 3, 199-209. (doi:10.1016/0376-6357(78)90013-X)

5. Schultner E, Pulliainen U. 2020 Brood recognition and discrimination in ants. Insect. Soc. 67, 1134. (doi:10.1007/s00040-019-00747-3)

6. Fénéron R, Jaisson P. 1995 Ontogeny of nestmate brood recognition in a primitive ant,Ectatomma tubercalutum Olivier (Ponerinae). Animal Behaviour 50, 9-14. (doi:10.1006/anbe.1995.0215)

7. Fouks B, d'Ettorre P, Nehring V. 2011 Brood adoption in the leaf-cutting ant Acromyrmex echinatior: Adaptation or recognition noise? Insectes Sociaux 58, 479-485. (doi:10.1007/s00040011-0167-9)

8. Madsen, Offenberg. 2017 Effect of pleometrosis and brood transplantation on colony growth of the black garden ant, Lasius niger. Asian Myrmecology 9, 1-11. (doi:10.20362/AM.009003)

9. Paul B, Annagiri S. 2019 Caught red-handed: behaviour of brood thieves in an Indian ant. Journal of Experimental Biology 222. (doi:10.1242/jeb.193755)

10. Lenoir A, d'Ettorre P, Errard C, Hefetz A. 2001 Chemical Ecology and Social Parasitism in Ants. Annual Review of Entomology 46, 573-599. (doi:10.1146/annurev.ento.46.1.573)

11. Buschinger A. 2009 Social parasitism among ants: A review (Hymenoptera: Formicidae). Myrmecological News 12, 219-235.

12. Satoi S, Iwasa Y. 2019 Chemical mimicry or crypsis - the evolutionary game played by parasitic ants invading other colonies. Theoretical Ecology , 1-9. (doi:10.1007/s12080-018-0406-z)

13. Grüter C, Jongepier E, Foitzik S. 2018 Insect societies fight back: the evolution of defensive traits against social parasites. Philosophical Transactions of the Royal Society B: Biological Sciences 373, 20170200. (doi:10.1098/rstb.2017.0200)

14. Sturgis SJ, Gordon DM. 2012 Nestmate recognition in ants (Hymenoptera: Formicidae): A review. Myrmecological News 16, 101-110.

15. van Zweden JS, d'Ettorre P. 2010 Nestmate recognition in social insects and the role of hydrocarbons. In Insect Hydrocarbons Biology, Biochemistry, and Chemical Ecology (eds GJ Blomquist, A-G Bagneres), pp. 222-243. Cambridge: Cambridge University Press. (doi:10.1017/CBO9780511711909.012) 
16. van Zweden JS, Brask JB, Christensen JH, Boomsma JJ, Linksvayer TA, d'Ettorre P. 2010 Blending of heritable recognition cues among ant nestmates creates distinct colony gestalt odours but prevents within-colony nepotism. Journal of Evolutionary Biology (doi:10.1111/j.14209101.2010.02020.x)

17. Liang D, Silverman J. 2000 "You are what you eat": Diet modifies cuticular hydrocarbons and nestmate recognition in the Argentine ant, Linepithema humile. Naturwissenschaften 87, 412416. (doi:10.1007/s001140050752)

18. Lenoir A, Depickère $S$, Devers $S$, Christidès J-P, Detrain C. 2009 Hydrocarbons in the Ant Lasius niger: From the Cuticle to the Nest and Home Range Marking. Journal of Chemical Ecology 35, 913-921. (doi:10.1007/s10886-009-9669-6)

19. d'Ettorre P, Heinze J, Ratnieks FLW. 2004 Worker policing by egg eating in the ponerine ant Pachycondyla inversa. Proceedings of the Royal Society of London. Series B: Biological Sciences 271, 1427-1434. (doi:10.1098/rspb.2004.2742)

20. Endler A, Liebig J, Schmitt T, Parker JE, Jones GR, Schreier P, Hölldobler B. 2004 Surface hydrocarbons of queen eggs regulate worker reproduction in a social insect. Proceedings of the National Academy of Sciences of the United States of America 101, 2945-2950. (doi:10.1073/pnas.0308447101)

21. Tannure-Nascimento IC, Nascimento FS, Dantas JO, Zucchi R. 2009 Decision rules for egg recognition are related to functional roles and chemical cues in the queenless ant Dinoponera quadriceps. Naturwissenschaften 96, 857-861. (doi:10.1007/s00114-009-0535-8)

22. van Zweden JS, Heinze J, Boomsma JJ, d'Ettorre P. 2009 Ant queen egg-marking signals: Matching deceptive laboratory simplicity with natural complexity. PLOS ONE 4, e4718. (doi:10.1371/journal.pone.0004718)

23. Holman L et al. 2010 Identification of an ant queen pheromone regulating worker sterility. Proceedings of the Royal Society B: Biological Sciences 277, 3793-3800. (doi:10.1098/rspb.2010.0984)

24. Ruel C, Lenoir A, Cerdá X, Boulay R. 2013 Surface lipids of queen-laid eggs do not regulate queen production in a fission-performing ant. Naturwissenschaften 100, 91-100. (doi:10.1007/s00114012-0997-y)

25. Helanterä $H$, d'Ettorre P. 2015 A comparative study of egg recognition signature mixtures in Formica ants. Evolution 69, 520-529. (doi:10.1111/evo.12590)

26. Schal C, Sevala VL, Young HP, Bachmann JAS. 1998 Sites of Synthesis and Transport Pathways of Insect Hydrocarbons: Cuticle and Ovary as Target Tissues. Am Zool 38, 382-393. (doi:10.1093/icb/38.2.382)

27. Schultner E, Oettler J, Helanterä H. 2017 The Role of Brood in Eusocial Hymenoptera. The Quarterly Review of Biology 92, 39-78. (doi:10.1086/690840)

28. d'Ettorre P, Tofilski A, Heinze J, Ratnieks FLW. 2006 Non-transferable signals on ant queen eggs. Naturwissenschaften 93, 136-140. (doi:10.1007/s00114-005-0075-9) 
29. Juárez MP, Fernández GC. 2007 Cuticular hydrocarbons of triatomines. Comparative Biochemistry and Physiology Part A: Molecular \& Integrative Physiology 147, 711-730. (doi:10.1016/j.cbpa.2006.08.031)

30. Howard RW, Blomquist GJ. 2004 Ecological, Behavioral, and Biochemical Aspects of Insect Hydrocarbons. Annual Review of Entomology 50, 371-393. (doi:10.1146/annurev.ento.50.071803.130359)

31. Villalta I, Amor F, Cerdá X, Boulay R. 2016 Social coercion of larval development in an ant species. The Science of Nature 103, 18. (doi:10.1007/s00114-016-1341-8)

32. Moreau CS, Bell CD, Vila R, Archibald SB, Pierce NE. 2006 Phylogeny of the Ants: Diversification in the Age of Angiosperms. Science 312, 101-104. (doi:10.1126/science.1124891)

33. Friard O, Gamba M. 2016 BORIS: a free, versatile open-source event-logging software for video/audio coding and live observations. Methods in Ecology and Evolution 7, 1325-1330. (doi:10.1111/2041-210X.12584)

34. RStudio Team. 2015 RStudio: Integrated Development Environment for R. Boston, MA: RStudio, Inc. See http://www.rstudio.com/.

35. R Core Team. 2019 R: A Language and Environment for Statistical Computing. Vienna, Austria: $\mathrm{R}$ Foundation for Statistical Computing. See https://www.R-project.org/.

36. van Zweden JS, Pontieri L, Pedersen JS. 2014 A statistical approach to identify candidate cues for nestmate recognition. Front. Ecol. Evol. 2. (doi:10.3389/fevo.2014.00073)

37. Ojala M, Garriga GC. 2010 Permutation tests for studying classifier performance. Journal of Machine Learning Research 11.

38. Koul A, Becchio C, Cavallo A. 2018 PredPsych: A toolbox for predictive machine learning-based approach in experimental psychology research. Behav Res 50, 1657-1672. (doi:10.3758/s13428017-0987-2)

39. Provost E, Riviere G, Roux M, Bagneres AG, Clement JL. 1994 Cuticular hydrocarbons whereby Messor barbarus ant workers putatively discriminate between monogynous and polygynous colonies. Are workers labeled by queens? Journal of Chemical Ecology 20, 2985-3003. (doi:10.1007/BF02098404)

40. Sharma KRR et al. 2015 Cuticular Hydrocarbon Pheromones for Social Behavior and Their Coding in the Ant Antenna. Cell Reports 12, 1261-1271. (doi:10.1016/j.celrep.2015.07.031)

41. Chernenko A, Helanterä H, Sundström L. 2011 Egg Recognition and Social Parasitism in Formica Ants. Ethology 117, 1081-1092. (doi:10.1111/j.1439-0310.2011.01972.x)

42. Helanterä H, Sundström L. 2007 Worker policing and nest mate recognition in the ant Formica fusca. Behavioral Ecology and Sociobiology 61, 1143-1149. (doi:10.1007/s00265-006-0327-5)

43. Pulliainen U, Helanterä H, Sundström L, Schultner E. 2019 The possible role of ant larvae in the defence against social parasites. Proceedings. Biological sciences 286, 20182867. (doi:10.1098/rspb.2018.2867) 
bioRxiv preprint doi: https://doi.org/10.1101/2021.03.17.435807; this version posted April 6, 2021. The copyright holder for this preprint (which

was not certified by peer review) is the author/funder, who has granted bioRxiv a license to display the preprint in perpetuity. It is made available under aCC-BY-NC-ND 4.0 International license.

444 44. Nascimento FS, Tannure-Nascimento IC, Dantas JO, Turatti IC, Lopes NP. 2013 Task-Related Variation of Cuticular Hydrocarbon Profiles Affect Nestmate Recognition in the Giant ant Dinoponera quadriceps. J Insect Behav 26, 212-222. (doi:10.1007/s10905-012-9353-5)

447

45. Rossi N, Baracchi D, Giurfa M, d'Ettorre P. 2018 Pheromone-Induced Accuracy of Nestmate Recognition in Carpenter Ants: Simultaneous Decrease in Type I and Type II Errors. The American Naturalist 193, 267-278. (doi:10.1086/701123) 


\section{$450 \quad$ Figure Legends}

451 Figure 1: Chemical profiles of egg and L1 larvae

452 A) Bar plots of the number of hydrocarbon compounds identified in egg and L1 larvae surface 453 extracts grouped by families: alkane (light grey), alkene (medium grey) and methyl-branched alkane 454 (m-alkane, dark grey). B) Boxplots of the total area of the compounds identified in hydrocarbon 455 compounds identified in egg and L1 larvae surface extracts grouped by families (same as in A). C) 456 Boxplots of the normalised area of the compounds identified in hydrocarbon compounds identified 457 in egg and L1 larvae surface extracts. The family of the compounds are displayed as in A.

460 A) Precisions of the linear discriminant analysis for each colony in each sample types performed from 461 the principal components, displayed in Supplementary Figure S3, that had an F-score superior or 462 equal to 0.01 . The black narrower lines represent the mean precision for each sample type. The red 463 wider line represents a random precision. Significance of the difference of mean precisions compared 464 to a random precision were computed with a permutation test. NS: $p \geq 0.05 ; *: p \leq 0.05 ; * *: p \leq$ $4650.01 ;{ }^{* * *}: \mathrm{p} \leq 0.001$. B) Ratios of the Euclidian distances between nestmate and non-nestmate measured with the global-centroid method from the principal components, displayed in $A$, that had an F-score superior or equal to 0.01 . Black dots represent outlier values that are 1.5 times outside the interquartile range. Letters represent groups of statistical similarity in each sample type (LMM ;

472 A) Boxplots of the number of nestmate (NM), non-nestmate (NNM) and hetero-specific (Mbar, Lnig or Ffus) eggs or larvae brought into the refuge by workers in all the behavioural trials. B) Boxplots of the total time spent by workers antennating brood during the trials where they displayed those behaviours. Diamonds represent the means. Letters show groups of statistical similarity in each

476 species (LMM ; Type II Anova ; $p \leq 0.05$ ). Black dots represent outlier values that are 1.5 times 477 outside the interquartile range. 


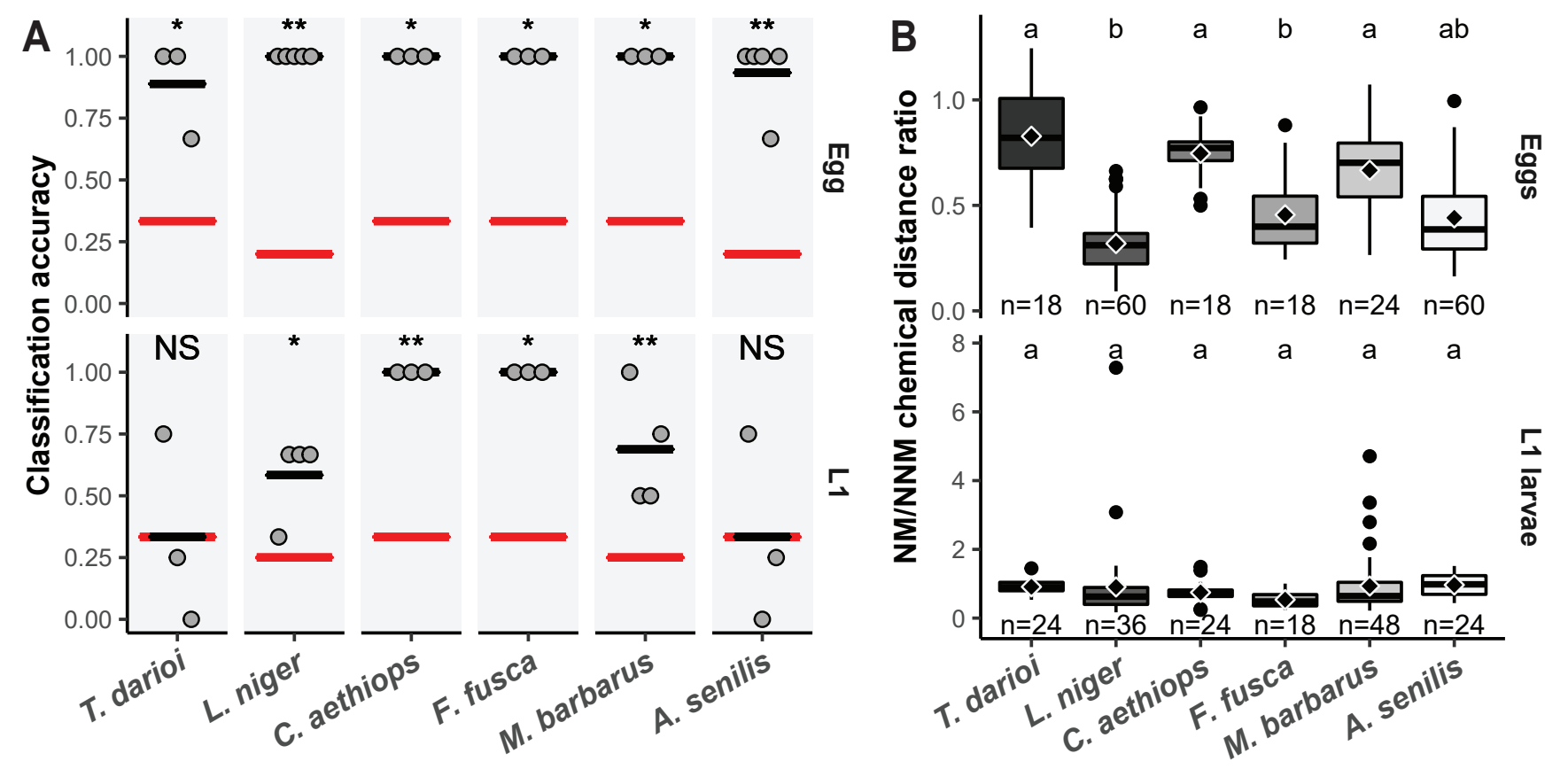


A
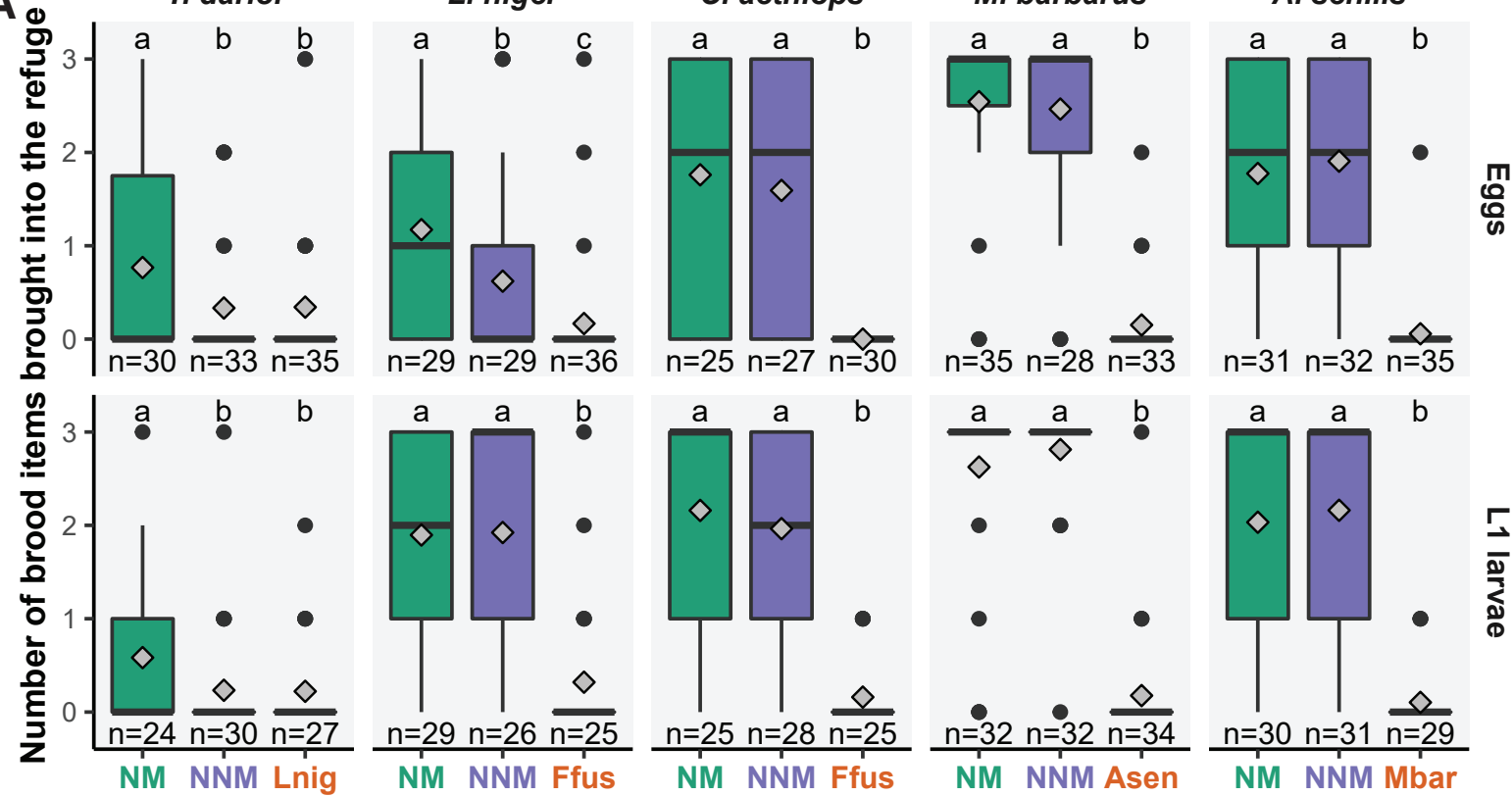

$n=35 n=28 \frac{\diamond}{n=33}$

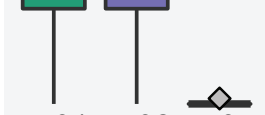

蒠

B

T. darioi

L. niger

C. aethiops

$\frac{a}{\diamond} \frac{a}{\diamond} \stackrel{b}{\circ}$

a $a$

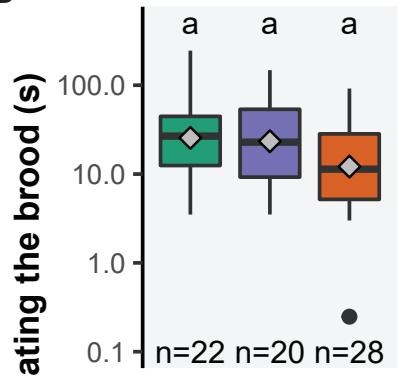

a $\quad$ a $a$

a $a \quad a$

M. barbarus

A. senilis

$\frac{n=32 \quad n=32 \frac{\diamond}{n=34}}{\text { NM NNM Asen }} \frac{n=30 \quad n=31 \frac{\diamond}{n=29}}{\text { NM NNM Mbar }}$
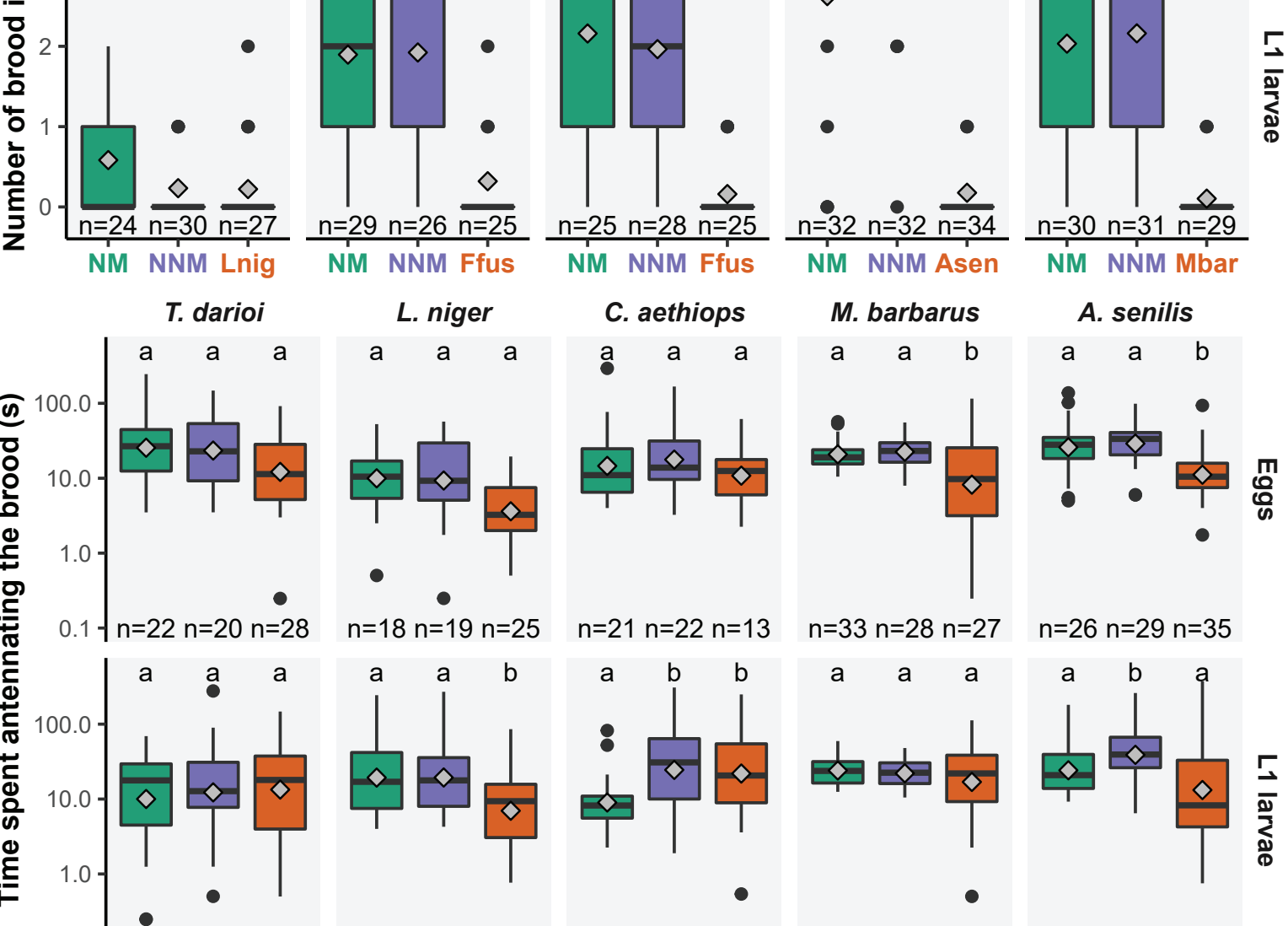

a $a \quad b$

$n=18 n=19 n=25$

$n=21 n=22 n=13$

$n=33 n=28 n=27$

$n=26 n=29 n=35$

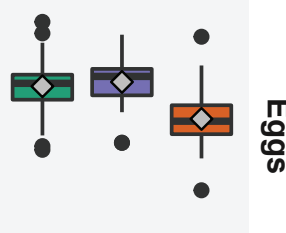

$0.1-n=18 n=23 n=25 \quad n=27 n=25 n=22$
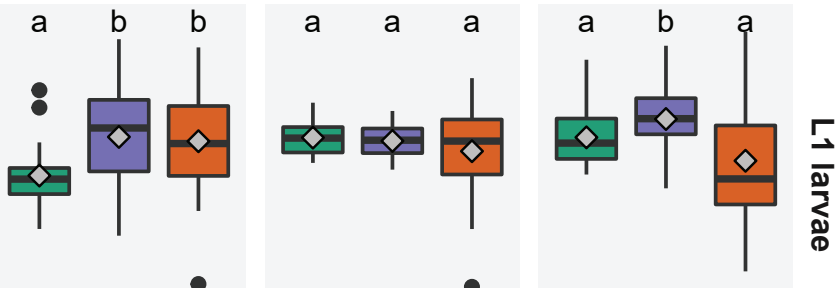
NM NNM Lnig

$\mathrm{n}=22 \mathrm{n}=25 \mathrm{n}=22$

$\frac{n=30 n=31 n=33}{\text { NM NNM Asen }} \frac{n=30 n=31 n=25}{\text { NM NNM Mbar }}$ 